\title{
Learning Classifier System Approach to Natural Language Grammar Induction
}

\author{
Olgierd Unold \\ Institute of Computer Engineering, Control and Robotics \\ Wroclaw University of Technology \\ Wyb. Wyspianskiego 27, 50-370 Wroclaw, Poland \\ olgierd.unold@pwr.wroc.pl \\ http://sprocket ict.pwr.wroc.pl/ unold
}

\begin{abstract}
This paper describes an evolutionary approach to the problem of inferring non-stochastic context-free grammar (CFG) from natural language (NL) corpora. The approach employs Grammar-based Classifier System (GCS). GCS is a new version of Learning Classifier Systems in which classifiers are represented by CFG in Chomsky Normal Form. GCS has been tested on the NL corpora, and it provided comparable results to the pure genetic induction approach, but in a significantly shorter time. The efficient implementation for grammar induction is very important during analysis of large text corpora.
\end{abstract}

\section{Introduction}

Syntactic processing, one of the complex task on natural language processing (NLP), has always been considered to be paramount to a wide range of applications, such as machine translation, information retrieval, speech recognition and the like. Historically, most computational systems for syntactic parsing, employ hand-written grammars, consisting of a laboriously crafted set of grammar rules to apply syntactic structure to a sentence. But in recent years, a lot of research efforts are trying to automatically induce workable grammars from annotated corpora. The process in which a system produces a grammar given a set of corpora is known as grammatical inference or grammar induction [4]. In general, the natural language (NL) corpora may contain both positive and negative examples from the language under study, which is described most often by context-free grammar (CFG). There are very strong negative results for the learnability of CFG. Effective algorithms exist only for regular languages, thus construction of algorithms that learn context-free grammar is critical and still open problem of grammar induction. Many researchers have attacked the problem of grammar induction by using evolutionary methods to evolve (stochastic) CFG or equivalent pushdown automata [8], but mostly for artificial languages like brackets, and palindromes. For surveys of the non-evolutionary approaches for CFG induction see [6].

In this paper we examine NL grammar induction using Grammar-based Classifier System (GCS) [7] - a new model of Learning Classifier System (LCS). In spite of intensive research into classifier systems in recent years [5] there is still slight number 
of attempts at evolving grammars using LCS. Bianchi in his work [2] revealed, on the basis of experiments with bracket grammars, palindromes and toy-grammar, higher efficiency of LCS in comparison with evolutionary approach. Cyre [3] inducted a grammar for subset of natural language using LCS but comparison to his results is hard since usage of corpora protected by trademarks. GCS tries to fill the gap also bringing grammar induction issues up. As was shown in [7], GCS achieves better results than Bianchi's system with reference to artificial grammars. This paper describes GCS approach to the problem of inferring non-stochastic CFG from NL corpora.

\section{Grammar-Based Classifier System}

The GCS operates similar to the classic LCS but differs from them in (i) representation of classifiers population, (ii) scheme of classifiers' matching to the environmental state, (iii) methods of exploring new classifiers. Population of classifiers has a form of a context-free grammar rule set in a Chomsky Normal Form (CNF). This is not a limitation actually because every CFG can be transformed into equivalent CNF. CNF allows only production rules in the form of $A \rightarrow \alpha$ or $A \rightarrow B C$, where $A, B, C$ are the non-terminal symbols and $a$ is a terminal symbol. The first rule is an instance of terminal rewriting rule. These ones are not affected by the genetic algorithm (GA), and are generated automatically as the system meets unknown (new) terminal symbol. Left hand side of the rule plays a role of classifier's action while the right side a classifier's condition. All classifiers (production rules) form a population of evolving individuals. In each cycle a fitness calculating algorithm evaluates a value (an adaptation) of each classifier and a discovery component operates only on a single classifier. CFG learns using a training set that consists of sentences both syntactically correct and incorrect. Grammar which accepts correct sentences and rejects incorrect ones is able to classify unseen so far sentences from a test set. Cocke-YoungerKasami parser $(\mathrm{CYK})$, which operates in $\Theta(\mathrm{n} 3)$ time, is used to parse sentences from corpus. Environment of classifier system is substituted by an array of CYK parser. Classifier system matches the rules according to the current environmental state (state of parsing) and generates an action (or set of actions in GCS) pushing the parsing process toward the complete derivation of the sentence analyzed. The discovery component in GCS is extended in comparison with standard LCS. In some cases a "covering" procedure may occur, adding some useful rules to the system. It adds productions that allow continuing of parsing in the current state of the system. Apart from the "covering" a GA also explores the space searching for new, better rules. Classifiers used in parsing positive examples gain highest fitness values, unused classifiers are placed in the middle while the classifiers that parse negative examples gain lowest possible fitness values. GCS uses a mutation of GA that chooses two parents in each cycle to produce two offspring. The selection step uses the roulette wheel selection. After selection a classical crossover or mutation can occur. Offspring that are created replace existing classifiers based on their similarity using crowding technique, which preserves diversity in the population and extends preservation of the dependencies between rules by replacing classifiers by the similar ones. 


\section{The Experiments}

Bianchi in [2] was not trying to use his system to induct a grammar for huge NL corpora. However such an experiment was performed using pure genetic algorithm and CFG by Aycinena at all [1]. Their system used grammar in CNF and a CYK parser, and as a corpora extensive part of various children books and the Brown linguistic data. The corpora were part-of-speech tagged using a Brill tagger. All English words were then removed - leaving only the tags themselves, and number of tags was reduced to 7 categories: nouns, pronouns; verbs, helping verbs; adjectives, numeral, possessives; adverbs; prepositions, particles; conjunctions, determiners; other (foreign words, symbols, and interjections).

The corpuses were divided into two parts, every third sentence was used for testing evolved grammar, and the remaining part of the corpora for inducing the grammars. The incorrect sentences were generated randomly from uniform distribution of length from 2 to 15 tags. Some comparison set of experiments with GCS was performed on the above NL corpora. Ten independent experiments were performed, evolution on each training corpus ran for 1,000 generations. The main results of the NL grammar induction with GCS are summarized in the table 1. In case of 5 corpuses the GCS model induced a grammar of higher quality fitness, for the brown this value is only slightly lower, and in the remaining 3 cases the estimator's value is lower, but not exceeding 5\%. The values of the positive estimator are in 8 cases significantly higher for the GCS model (the differences oscillate in the range of $4.2 \%$ and $16.2 \%$ ), and for the brown corpus the AKM approach got a result which is better by $0.5 \%$. Undoubtedly, the worst for the GCS model comes up the comparison of the negative values - for each corpus the model got decidedly higher values of this estimator, and the differences oscillate in the range $1 \%$ for wizard to $17.3 \%$ for tom corpus. It indicates that during the grammar induction the GCS model created in a few cases (for 5 bodies the differences do not exceed 7\%) productions which are too universal in comparison to the AKM approach, which also parse a part of negative sentences. The last parameter which can be compared is the number of evolutionary steps (evals), in which both approaches found their best solutions. In as many as 6 cases the GCS model did not exceed 50 steps, in the next case did not exceed 100 steps, and two longest inductions took only slightly above 500 steps (somewhat over an hour). The AKM approach took, in the best case, 15,500 steps, and for as many as 5 corpora - 200,000 steps, and, according to the authors, 60 hours of calculation! The GCS model proved to be incomparably more effective, being able to find, in the majority of cases, the grammars with higher values of fitness and positive estimators. The evolved grammar learned for the corpus children indicates some interesting linguistic features. There are quite obvious groups like adjective noun, as well as rule noun verb. The model found in the corpus also often appearing in English bigrams, so as noun adverb, noun conjunction, verb adverb, or verb conjunction. The sentence can start from the article why adding the article for the beginning of sentence is also keeping its correctness. The straight majority of context-free production rules are beginning from the starting symbol what is suggesting the big generality of these rules. On one hand it will knock for economical writing entire grammar on the other however such a versatility is enabling parsing also of sentences not belonging to the language. 
Table 1. Comparison of NL grammar induction using genetic approach (AKM) with GCS. The corpora include a selection of children's books (denoted children, 986 learning correct sentences, and 986 learning incorrect sentences), The Wizard of Oz (wizard, 1540/1540), Alice in Wonderland (alice, 1012/1012), Tom Sawyer (tom, 3601/3601), and five Brown corpora: brown_a (2789/2789), brown_b(1780/1780), brown_c (1099/1099), brown_d (1062/1062), and brown_e (2511/2511). For each learning corpus, the table shows the target language, and four sets of results. The first is the best fitness gained by GCS within 10 experiments and compared approach. The fitness describes the percentage of sentences (correct and incorrect) recognized correctly. Next results of the GCS model refer to the experiment in which best fitness was obtained. The second result, positive, shows the percentage of correct examples from the train set classified correctly. The third sort of results, negative, is the percentage of negative examples classified incorrectly, and the last one indicates the number of generations needed to reach the best fitness (evals).

\begin{tabular}{|c|c|c|c|c|c|c|c|c|}
\hline \multirow{2}{*}{ Corpus } & \multicolumn{2}{|c|}{ fitness } & \multicolumn{2}{c|}{ positive } & \multicolumn{2}{c|}{ negative } & \multicolumn{2}{c|}{ evals } \\
\cline { 2 - 9 } & GCS & AKM & GCS & AKM & GCS & AKM & GCS & AKM \\
\hline children & 93,2 & 93,1 & 98,8 & 91,8 & 12,5 & 5,7 & 9 & 200,000 \\
\hline wizard & 94,6 & 90,2 & 99,3 & 89,5 & 10,2 & 9,2 & 32 & 200,000 \\
\hline alice & 89,5 & 92,1 & 96,8 & 92,5 & 17,9 & 8,4 & 81 & 200,000 \\
\hline tom & 86,3 & 92,1 & 98,4 & 92,7 & 25,9 & 8,6 & 3 & 200,000 \\
\hline brown_a & 93,8 & 94,0 & 98,3 & 94,1 & 11,6 & 6,1 & 45 & 48,500 \\
\hline brown_b & 94,6 & 94,0 & 99,3 & 94,7 & 10,2 & 6,7 & 506 & 200,000 \\
\hline brown_c & 92,5 & 87,9 & 96,7 & 80,5 & 11,7 & 4,7 & 592 & 15,500 \\
\hline brown_d & 91,6 & 91,3 & 97,1 & 88,2 & 13,8 & 5,6 & 18 & 45,000 \\
\hline brown_e & 89,5 & 94 & 93,4 & 93,9 & 14,5 & 5,9 & 38 & 122,000 \\
\hline
\end{tabular}

\section{References}

1. Aycinena, M., Kochenderfer, M.J., Mulford D.C.: An evolutionary approach to natural language grammar induction. Final project for CS224N: Natural Language Processing. Stanford University (2003)

2. Bianchi, D.: Learning Grammatical Rules from Examples Using a Credit Assignement Algorithm. In: Proc. of The First Online Workshop on Soft Computing (WSC1), Nagoya (1996) 113-118

3. Cyre, W.R.: Learning Grammars with a Modified Classifier System. In: Proc. 2002 World Congress on Computational Intelligence, Honolulu Hawaii (2002) 1366-1371

4. Gold, E.: Language identification in the limit. Information Control 10 (1967) 447-474

5. Lanzi, P.L., Riolo, R.L.: A Roadmap to the Last Decade of Learning Classifier System Research. LNAI 1813, Springer Verlag (2000) 33-62

6. Lee, L.: Learning of Context-Free Languages: A Survey of the Literature. Report TR-12-96. Harvard University, Cambridge, Massachusetts (1996)

7. Unold, O.: Playing a toy-grammar with GCS. In Mira J., Álvarez J.R. (eds.) IWINAC 2005, LNCS 3562 (2005) 300-309

8. Unold, O.: Context-free grammar induction with grammar-based classifier system. Archives of Control Science, vol. 15 (LI) 4 (2005) 681-690 